\title{
Notes sur les Finances Publiques
}

\author{
M. Barry Nouhoum Aly
}

Les finances publiques sont l'étude des règles et des opérations relatives aux deniers publics. Selon le critère organique, les finances publiques peuvent aussi être présentées comme l'ensemble des règles gouvernant les finances de l'Etat, des collectivités territoriales, des organismes de sécurité sociale, des établissements publics et de toutes autres personnes morales de droit public. C'est un champ à la croisée du droit fiscal, du droit constitutionnel, ainsi que de la comptabilité publique.

L'originalité des finances publiques repose sur 5 centres d'intérêts :

Objet financier, Etude de l'organisation financière des pouvoirs publics (ex : Ministère des Finances) et également les activités financières des pouvoirs publics (des administrations publiques : Douanes, Impôts, Trésor, Domaines). Mais les finances publiques se différencient des finances privées ou des finances générales par les finalités poursuivies et les moyens utilisés. Les finalités des finances publiques consistent en effet, à satisfaire/contribuer les besoins d'intérêt général.

Les prélèvements obligatoires concernent les impôts + cotisations sociales.

Les moyens juridiques sont très originaux. Les personnes publiques disposent de prérogatives de puissance publique, de prérogatives exorbitantes du droit commun aussi dans l'exercice de leurs activités financières. Dont la plus significative est celle que l'on appelle le privilège du préalable, que l'on appelle également le pouvoir ou la voie d'exécution d'office des décisions administratives (ex : En cas d'opposition du contribuable pour payer son impôt, l'administration va utiliser d'elle-même les procédures de recouvrement d'office/ forcées sans passer par un juge suivant des privilèges dévolus à des administrations financières.

\section{Objet économique}

Les budgets publics ont un objet et un effet économique. En effet, les politiques budgétaires font partie/participent de la politique économique des Etats, aux côtés des politiques monétaires ou des interventions juridiques. L'instrument budgétaire est utilisé, par exemple, pour relancer l'économie, c.à.d. une politique de la demande qui consiste à augmenter massivement les dépenses publiques.

\section{Objet sociologique}

Les finances publiques participent de la science politique et de la science administrative.

Elles participent de la science politique car l'approche financière publique révèle parfaitement la réalité de l'organisation, de la distribution et du fonctionnement du pouvoir po- 
litique. Par exemple, qui décide les impôts et les dépenses publiques. Est-ce le pouvoir exécutif ou le Parlement?

Principe de libre administration, $\Rightarrow$ autonomie financière des collectivités territoriales. Sujétion très forte des collectivités territoriales à l'Etat.

\section{Objet juridique}

Les activités financières publiques font l'objet d'un fort encadrement juridique, pour deux raisons :

- Raison politique : Pour protéger le pouvoir financier des Assemblées délibérantes.

- Raison technique : Pour protéger les fonds publics.

Il en résulte un droit financier public de 4 branches :

- Règles générales

- LE DROIT BUDGETAIRE : Ensemble des règles relatives à la préparation, au conte$\mathrm{nu}$, à l'adoption et au contrôle des budgets publics.

- LE DROIT DE LA COMPTABILITE PUBLIQUE : Ensemble des règles juridiques relatives à l'exécution des opérations financières des personnes publiques et à leur contrôle. Décret portant Réglementation Générale sur la Comptabilité Publique (RGCP)

- Règles spécialisées

- LE DROIT FISCAL : Ensemble des règles juridiques relatives au régime des impôts et à la procédure de leur recouvrement. Relève de la compétence du législateur.

- LE DROIT DES MARCHES PUBLICS : Ensemble des règles juridiques relatives à la commande publique, c.à.d. la commande publique pour la réalisation des achats et des opérations, en matière de fournitures, de travaux et de services. Formalisé par la Code des Marchés Publics.

\section{Objet gestionnaire}

Les Etats doivent optimiser les moyens financiers de façon à conserver le même train de vie, voire améliorer la qualité des services publics.

\section{Loi des Finances ou budget d'Etat}

Le budget est défini comme étant « l'acte par lequel sont prévues et autorisées les recettes et dépenses annuelles de l'Etat ou des autres services que les lois assujettissent aux mêmes règles. 
A cet égard 4 points sont à relever :

- Le budget est un acte juridique, qui émane d'une institution le parlement,

- Le budget est un état prévisionnel : les recettes et les dépenses sont considérées comme probables et évaluées par anticipation

- Le budget est acte d'autorisation : l'acte qui émane de l'Assemblée Nationale ou du Parlement engage l'exécutif à procéder à son exécution.

- Cet acte juridique est annuel, dès lors, cette autorisation n'est valable que pour une année.

Au total, le budget est l'état de prévisions des recettes et des dépenses d'une période donnée et dont la réalisation est autorisée par la loi des finances, acte-condition. Celle-ci fixe les voies et moyens pour l'ensemble à savoir les ressources et la façon dont elles seront réalisées.

A travers ces définitions, il importe de reconnaître à l'institution compétente la vocation d'habilitation d'une et d'autre part l'établissement d'un document comptable chiffré décrivant les recettes et les dépenses de l'Etat.

Le budget est préparé et adopté suivant un certain nombre de principes :

Les principes en matière de recette publique, retiennent les 4 piliers de la fiscalité moderne :

- Nécessité de l'impôt,

- Généralité de l'impôt,

- Consentement à l'impôt,

- Egalité devant l'impôt.

Comme autre série de principes, il faut noter entre autres :

- l'autorisation de suivre l'emploi des contributions, c'est-à-dire décidé des dépenses publiques.

- le contrôle des finances publiques qui s'exerce a priori et postériori de l'exécution de la loi des finances. Ce contrôle est soit administratif, parlementaire ou judiciaire.

Le droit budgétaire avec les principes budgétaires. Grands principes budgétaires :

- Annualité,

- Unité budgétaire,

- Spécialité budgétaire,

- Universalité budgétaire.

Le droit de la comptabilité publique, structure les grands principes de la comptabilité publique :

- Séparation des ordonnateurs et des comptables,

- Responsabilité des gestionnaires de fonds publics, plus précisément le principe de la responsabilité des comptables publics.

- Unité de trésorerie qui veut que tous les fonds des organismes publics soient centralisés sur le compte courant du Trésor. 
Au Mali, avant l'avènement d'un régime financier harmonisé de l'UEMOA, le budget traditionnel était un budget de moyens règlementé par les textes suivants :

- La loi 96-060 portant loi des finances,

- La loi 96-061 portant principes fondamentaux de lacomptabilité publique

- Décret n 97-192 PRM, portant règlement de la comptabilité publique

Selon ce procédé, la performance se limitait au respect des lois et règlements en vigueur et au contrôle des moyens.

C'est seulement en 2018 que le Mali a basculé au budget au mode programme conformément aux directives UEMOA qui ont été transposées dans la législation malienne et qui retient dans les critères de performance l'efficacité, l'économie et l'efficience des programmes et activités selon des indicateurs établis.

A ce jour, les textes régissant les finances publiques au Mali sont retracés dans le tableau ci-dessous :

- la Constitution du Mali;

- le cadre harmonisé de la gestion des finances publiques au sein des pays de l'UEMOA transposés dans la législation nationale comme suit :

\begin{tabular}{|c|c|}
\hline Libellé directive & $\begin{array}{c}\text { Texte de transposition dans la législation } \\
\text { nationale }\end{array}$ \\
\hline $\begin{array}{l}\text { Directive } n^{\circ} 1 / 2009 / \mathrm{CM} / \text { UEMOA portant code } \\
\text { de transparence dans la gestion des finances }\end{array}$ & $\begin{array}{l}\text { Loi n }{ }^{\circ} 2013-031 \text { du } 23 \text { juillet } 2013 \text { portant ap- } \\
\text { probation du code de Transparence dans la Ges- } \\
\text { tion des Finances Publiques }\end{array}$ \\
\hline $\begin{array}{l}\text { Directive } \mathrm{N}^{\circ} 06 / 2009 / \mathrm{CM} / \mathrm{UEMOA} \text { portant lois } \\
\text { de finances au sein de l'UEMOA }\end{array}$ & $\begin{array}{l}\text { Loi n²013 -028 du } 11 \text { juillet 2013, modifiée, re- } \\
\text { lative aux lois de finances }\end{array}$ \\
\hline $\begin{array}{l}\text { Directive } \mathrm{N}^{\circ} 07 / 2009 / \mathrm{CM} / \mathrm{UEMOA} \text { portant rè- } \\
\text { glement général sur la comptabilité publique au } \\
\text { sein de l'UEMOA }\end{array}$ & $\begin{array}{l}\text { Décret n²014-349/ P-RM du } 22 \text { Mai } 2014 \text { por- } \\
\text { tant règlement général sur la comptabilité pu- } \\
\text { blique }\end{array}$ \\
\hline $\begin{array}{l}\text { Directive } \mathrm{N}^{\circ} 08 / 2009 / \mathrm{CM} / \mathrm{UEMOA} \text { portant no- } \\
\text { menclature budgétaire de l'Etat au sein de l'UE- } \\
\text { MOA }\end{array}$ & $\begin{array}{l}\text { Décret n²014-0694/P-RM du } 12 \text { septembre } \\
2014 \text { portant nomenclature budgétaire de l'Etat }\end{array}$ \\
\hline $\begin{array}{l}\text { Directive } \mathrm{N}^{\circ} 09 / 2009 / \mathrm{CM} / \mathrm{UEMOA} \text { portant plan } \\
\text { comptable de l'Etat au sein de l'UEMOA }\end{array}$ & $\begin{array}{l}\text { Décret n`2014-0774/P-RM du } 14 \text { octobre } 2014 \\
\text { portant plan comptable de l'Etat }\end{array}$ \\
\hline $\begin{array}{l}\text { Directive } \mathrm{N}^{\circ} 10 / 2009 / \mathrm{CM} / \mathrm{UEMOA} \text { portant ta- } \\
\text { bleau des opérations financières de l'Etat au sein } \\
\text { de l'UEMOA }\end{array}$ & $\begin{array}{l}\text { Décret n`2014-350/ P-RM du } 22 \text { Mai } 2014 \text { por- } \\
\text { tant tableau des opérations financières de l'Etat }\end{array}$ \\
\hline
\end{tabular}

- la loi № 2014-049 du 19 septembre 2014, portant principes fondamentaux de la création, de l'organisation, de la gestion et du contrôle des services publics; 
- le Décret n²015-0604/PRM du 25 Septembre 2015 portant code des marchés publics et des délégations de services publics;

- le décret $\mathrm{n}^{\circ} 10-681 / \mathrm{PRM}$ du 30 décembre 2010 portant réglementation de la comptabilité-matières

NB : le Décret n²014-349/ P-RM du 22 Mai 2014 portant règlement général sur la comptabilité, a été révisé par le Décret 2018-0009 P-RM Le Règlement Général de la comptabilité publique.

Cependant, les finances publiques maliennes doivent relever d'énormes défis qui sont entre autres :

\section{DEFIS DE LA MODERNISATION DES PROCEDURES ET DES INSTRUMENTS}

Il s'agit de :

- Renforcer le système d'informatisation (préparation et exécution de la loi de finance et de reddition des comptes);

- Assurer la fiabilité, la transparence et l'exhaustivité de données sur les finances publiques (préparation et exécution);

- Améliorer et sécuriser la connexion informatique de tous les systèmes centraux et régionaux;

- Développer la pratique des contrats plans avec tous les établissements publics;

\section{DEFIS DE LA MOBILISATION DES RESSOURCES INTERNES ET EXTERNES}

- Il s'agit de maîtriser les exonérations físcales;

- Améliorer la gestion de la trésorerie en tenant compte du profil de l'exécution budgétaire et des différentes natures de dépenses;

- Renforcer l'unicité des caisses du trésor à travers la limitation des comptes dans les banques commerciale, les comptes d'affectation spéciales et les affectations dans les budgets autonomes;

\section{LES DEFIS DEMOGRAPHIQUES}

- Selon toutes les études disponibles, la population malienne est assurée de doubler durant les prochains 25 ans même dans le cas d'une hypothèse basse de la fécondité.

La population malienne croit de 3,6\% par an.

Il faut noter que pour relever ce défi démographique, il faut nécessairement améliorer la croissance économique et le financement; 


\section{LES DEFIS DE LA REFORME DE L'ETAT ET DE LA QUALITE DES RESSOURCES HUMAINES DES RESSOURCES HUMAINES}

En dix ans l'administration malienne a fortement évolué :

- Le nombre des unités administratives constituant des Le nombre des unités administratives constituant des centres d'allocation budgétaires est passé de 1031 en 2000 à 2963 en 2011

- Le nombre des administrations centrales est passé de 109 Le nombre des administrations centrales est passé de 109 en 2000 à 246 en 2011.

- Le nombre des EPA et assimilés est passé de 31 en 2000 à 1342011134 en 2011.

- Le nombre des services régionaux est passé de 54 à 733 en 2011

\section{LES DEFIS DE LA REFORME DE L'ETAT}

Relire les textes fondamentaux relatifs à la création à l'organisation et aux modalités de fonctionnement des services publics pour les rendre cohérent avec les

Directives de l'UEMOA 1 directives de l'UEMOA en prenant en compte la dimension « responsable de programmes »;

- Nécessité de corréler les réformes institutionnelles et administratives avec la souténabilité budgétaire en mettant en exergue les seuls critères d'efficacité et d'efficience des réformes;

- Réformer l'Etat sur la base des audits fondés principalement sur les fonctions que sur les besoins de développement isolés des administrations : ceci permettra développement isolés des administrations : ceci permettra de redimensionner les administrations dans un objectif d'efficience et d'efficacité;

- Améliorer le processus de la décentralisation en instituant Améliorer le processus de la décentralisation en instituant les contrats plans entre l'Etat et les collectivités

\section{LES DEFIS DE LA RATIONALISATION DU CONTROLE}

Se fondant sur les acquis dans le domaine de l'informatisation de la chaîne de la dépense et des efforts en cours dans le cadre de l'institutionnalisation de la gestion axée sur les Résultats et à l'instar des pays développés et en développement qui sont dans ce processus une rationalisation du contrôle s'impose pour éviter les redondances qui entravent l'efficacité des dépenses. Dans la perspective de la mise en place du nouveau cadre harmonisé des finances publiques une mutation du contrôle est aujourd'hui indispensable en passant du contrôle de régularité vers un contrôle de gestion dans le contrôle de régularité vers un contrôle de gestion dans le sens de l'efficacité et de la performance en mettant l'accent sur l'audit interne. 
Renforcer le cadre institutionnel de suivi des recommandations des missions de contrôle et de

Vérification. Et en fin

\section{LES DEFI SDE L'ACCES DU PUBLIC A L'INFORMATION BUDGETAIRE}

- Développer des annexes thématiques à la loi de finances

- Mettre sur le site internet toutes les informations relatives au budget:

- Lettre de cadrage Lettre de cadrage

- Les lois de finances et leurs annexes

- Les situations d'exécution infra annuelles

- Les Arrêtés d'ouverture de crédit

- Les Arrêtés sur les transferts et virements de crédit en cours d en cours d'année

- Le rapport trimestriel d'exécution de la loi de finances

- Elaboration de documents budgétaires spéciaux plus accessibles au public.

MERCI DE VOTRE AIMABLE ATTENTION 